\begin{tabular}{|c|l|}
\hline Title & The Optimal Caste Ratio in Polymorphic A nts: Estimation and Empirical Evidence \\
\hline Author(s) & Hasegawa, Eisuke \\
\hline Citation & $\begin{array}{l}\text { The A merican Naturalist, 149(4), 706-722 } \\
\text { https://doi.org/10.1086/286016 }\end{array}$ \\
\hline Issue Date & 1997-04 \\
\hline Doc URL & http://hdl.handle.net/2115/66645 \\
\hline Type & article \\
\hline File Information & 286016.pdf \\
\hline
\end{tabular}

Instructions for use 


\title{
THE OPTIMAL CASTE RATIO IN POLYMORPHIC ANTS: ESTIMATION AND EMPIRICAL EVIDENCE
}

\author{
Eisuke Hasegawa* \\ Center for Ecological Research, Kyoto University, Kyoto 606-01, Japan \\ Submitted February 22, 1996; Revised July 10, 1996; Accepted July 12, 1996
}

\begin{abstract}
A simple model is proposed for estimation of the optimal caste composition in polymorphic ants. The model gives the optimal caste composition as the highest point on a hypersurface that represents the total efficiency of the colony as a whole. The hypersurface is the product of all partial efficiency surfaces for all tasks. The model was applied to the dimorphic ant Colobopsis nipponicus to test its utility. The ergonomic efficiency of the colony as a whole was estimated from the quantitative ability of each caste to perform each role. When all partial functions of the major workers were included in the model, the predicted optimal ratio closely reflects the observed caste ratio in the field. In addition, the pattern of changes in brood productivity upon experimental manipulation of the caste ratio was consistent with the pattern predicted from the model. These results suggest that the model is useful for estimations of the optimal caste ratio and that the caste ratio in field colonies is tuned to maximize the ergonomic efficiency of the colony as a whole through colony-level selection.
\end{abstract}

Worker polymorphism is a prominent characteristic of ants, which are eusocial hymenopteran insects (Wilson 1971; Hölldobler and Wilson 1990). Wilson (1953) classified the polymorphism of ant workers into several categories by applying allometric criteria, and he provided a model for the evolution of worker polymorphism in ants. In his classification, complete dimorphism with two distinct subcastes is one of the most advanced stages.

Division of labor, based on morphology, is common in polymorphic ants, and some castes specialize in specific tasks (Wilson 1976, 1978, 1980a, 1980b; Cole 1980; Mirenda and Vinson 1981; Busher et al. 1985; Moffet 1986, 1987; Patel 1990; Tsuji 1990; Villet 1990; Detrain and Pasteels 1992; Lachaud et al. 1992). Major workers specialize in a few specific tasks, such as nest defense (Wilson 1976; Detrain and Pasteels 1992; Hasegawa 1993b), food milling (Wilson 1978), and food storage (Wilson 1974; Tsuji 1990; Lachaud et al. 1992; Hasegawa 1993a). By contrast, minor workers generally engage in daily tasks in the colony, such as brood care, queen(s) care, foraging, and nest maintenance. In addition, division of labor is based not only on morphology but also on the age of individuals, namely, age polyethism (see Hölldobler and Wilson 1990).

When two subcastes of workers exist in a colony, there is necessarily a ratio

\footnotetext{
* Present address: Laboratory of Wildlife Conservation, National Institute for Environmental Studies, Onogawa 116-2, Tsukuba, Ibaraki 305, Japan; E-mail: ehase@nies.go.jp. 
between the numbers of members of the two subcastes. Because each subcaste differentiates functionally, different caste ratios must result in differences in ergonomic efficiency of the colony as a whole. Oster and Wilson (1978) argued that the ratio between functionally differentiated castes should be tuned to maximize the ergonomic efficiency of the colony as a whole. Moreover, selection that shapes caste ratios would act on the colony as a whole. They proposed a theoretical model for estimation of the optimal caste ratio in a colony. However, the model has proved difficult to apply to the evaluation of empirical data.

Although many studies of division of labor have been done (see previously cited references), only a few have focused on the relationships between the roles of different subcastes and the caste ratio (Porter and Tschinkel 1985; Walker and Stamps 1986; Fowler 1987; Tsuji 1994). Indeed, no estimations of the optimal caste ratio have ever been made, probably as a consequence of the complexity of the previously proposed models. In addition, this study area is disappearing from the scientific field, although some data suggest that natural selection is working on the overindividual level. Roles of selection on the overindividual level is an untouched area in evolutionary biology. For example, Porter and Tschinkel (1985) and Tsuji (1994) reported that the brood productivity of the colony as a whole was affected by morphology or an age-based subcaste ratio, and their studies suggested the influence of colony-level selection on caste ratio. Thus, caste ratio studies are especially important when we try to understand the hierarchical selection structures that operate in insect societies, in particular, and in social groups of organisms, in general.

I have previously investigated the roles of the two subcastes in the dimorphic ant Colobopsis nipponicus in quantitatively analyses (Hasegawa 1993a, 1993b). In this study, I propose a simple model for estimation of the optimal caste composition in polymorphic ants. The model is applied to the dimorphic species $C$. nipponicus, by reference to the quantitative efficiencies of each caste in the performance of each role. Caste ratios observed in the field are compared with the estimated ratios to determine whether the estimates are concordant with empirical data. In additional experiments, the effects of caste ratios on brood productivity were examined by manipulation of caste ratios in experimental colonies.

\section{THE MODEL}

In this section, a general model is proposed for a polymorphic species that has more than two subcastes. The model is then explained in more detail in the case of dimorphic species.

If there is more than one class of workers (= subcaste) in a colony and if each class specializes in different tasks to a different specific extent, there must be an optimal caste composition that maximizes the ergonomic efficiency of the colony as a whole. How do we find the optimal caste composition (the relative proportion of each caste)? A general approach is as follows. When there are $n$ subcastes within a colony, any caste composition can be represented as a point on a hypersurface in a multidimensional space with $n-1$ dimensions. Each axis ranges 
from 0 to 1 because each axis represents the relative proportion of each subcaste. The hypersurface mentioned earlier is defined as a surface on which any point satisfies the equation $p_{1}+p_{2}+\ldots+p_{n}=1$, where $p_{i}$ is the proportion of the $i$ th subcaste in the total population of workers; it is called a caste surface hereafter. If we define an efficiency axis (ranging from 0 to 1 ) that intersects the caste surface vertically, the efficiency function of the colony for a given task can be represented as a hypersurface that covers the caste surface. If there are $t$ tasks, there are $t$ hypersurfaces over the caste surface. Then, the total ergonomic efficiency at a given point on the caste surface is obtained by multiplying the values of all $t$ efficiency surfaces at that point. The total efficiency function of the colony as a whole is obtained as a synthesized hypersurface composed of all the efficiency surfaces over the caste surface. Now, the optimal caste composition can be obtained as a point that gives the maximum efficiency on the total efficiency surface. In a later section, this construct is proved mathematically for a dimorphic species.

The model is explained in more detail for a hypothetical dimorphic species. When gain of fitness through each task is standardized by reference to the maximum fitness from a task that possibly achieves the highest gain in fitness among all tasks, the fitness gain of each task can be represented as a caste ratio function that varies between 0 and 1 . Such functions represent the pattern of changes with caste ratio in the colony's efficiency for performance of each task. For example, when there are two tasks and each task contributes equally to fitness gain, each efficiency function has the same maximum value of 1 (fig. $1 A$ ). If each different task contributes unequally to fitness gain, the efficiency function for the most efficient task has the highest value $(=1)$, and the other efficiency function has a maximum value that is $<1$ (see fig. $1 B$ ). However, we never know the actual maximum values of each function because the observed fitness is the result of the total efficiency function. In such cases, we can calculate the total efficiency function by assuming that all the partial functions have the same maximum value (see the next section). Thus, the maximum value of the efficiency functions that has a lower maximum value can be scaled up to the maximum value of 1 (see fig. $1 B$ ). In figure $1 A$ and $B$, the efficiency for task $A$ (represented as $t_{\mathrm{A}}[r]$ ) and that for task $\mathrm{B}\left(t_{\mathrm{B}}[r]\right)$ change linearly with caste ratio. This pattern is predicted when

FIg. 1.-Graphic illustration of the concepts of the model for estimation of the optimal caste ratio $(O C R)$ in dimorphic social insects. Increasing and decreasing lines with caste ratio represent changes in colony efficiency through tasks A and B, respectively, to which two castes are specialized to different extents. In all cases, the total efficiency function (convex curves) of the colony is represented as the product of the efficiency values of all partial functions. Figure $1 A$ shows a case in which both castes are highly specialized in their roles, which contribute equally to colony fitness. A case of the unequal contribution of each role to colony fitness is shown in figure $1 B$. The increasing line with a gentle slope in figure $1 B$ shows the actual relative contribution to colony fitness through task A. A scaled-up version of this line (increasing line with a steep slope) can be used in calculations of the optimal caste ratio (for proof, see text). Figure $1 C$ shows a case of a nonlinear pattern of change in colony efficiency that occurs when the size between castes is very different or when the effects of the caste ratio on task efficiency are not additive. 

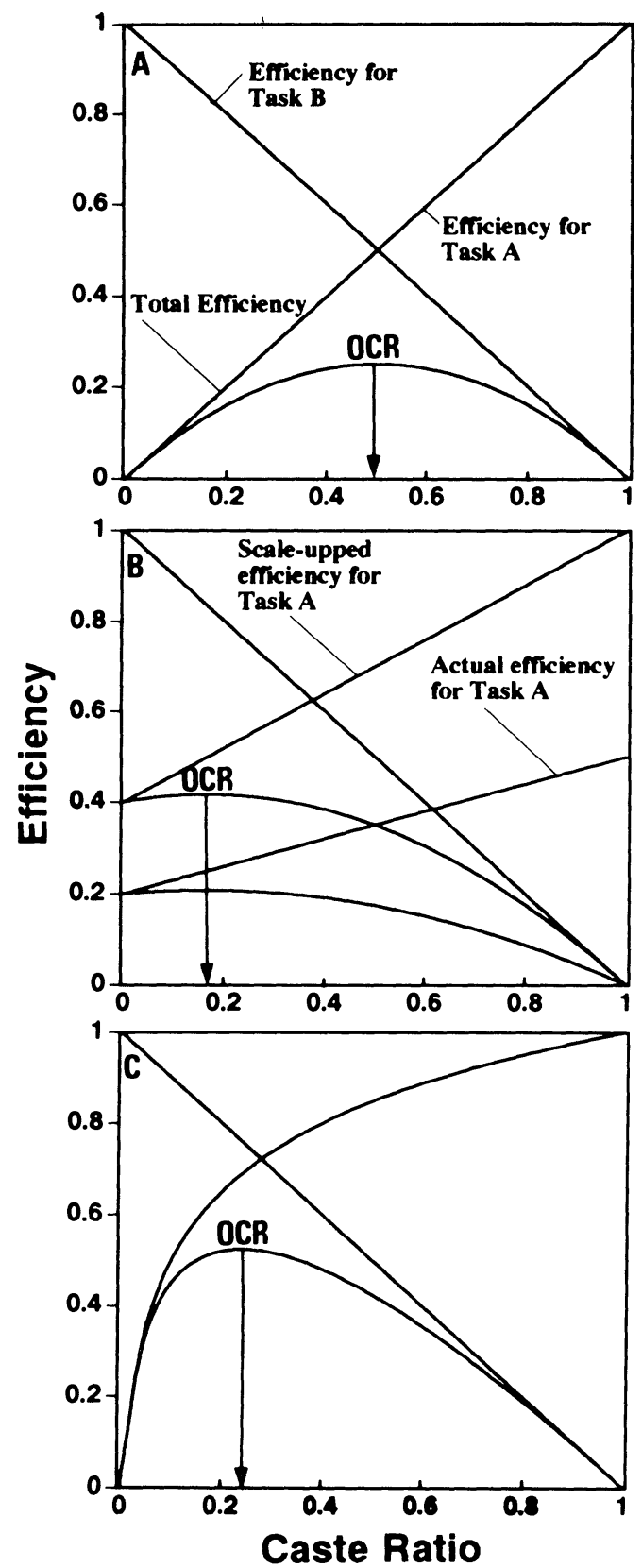
the size (= production cost) of the two subcastes is the same and when each subcaste contributes quantitatively to their roles. However, in some case, the efficiency for some tasks changes nonlinearly. Figure $1 C$ shows an example of such a case. This pattern is predicted when there is a large difference in size between the castes or when the castes contribute qualitatively to their roles. However, in all cases, the total efficiency function can be represented by the locus of the product of the two efficiencies (the convex curves in fig. $1 A-C$ ), and the optimal ratio is the ratio that yields the maximum fitness gain of the colony through performance of the both tasks.

Let us consider mathematically whether this argument holds for any shape and relative height of the efficiency functions. If there are $t$ tasks in a colony, it can be said that the total colony efficiency at the ratio $r$ is represented by $T(r)=$ $\Pi \alpha_{i} t_{i}(r)$, where $\alpha_{i}$ is a constant $\left(0<\alpha_{i} \leq 1\right)$ by which the efficiency or performance of each task is weighted to the maximum fitness achieved through the most efficient task, and $t_{i}(r)$ is the efficiency function for the $i$ th task. If all partial tasks contribute equally to the production of reproductives, all $\alpha_{i}=1$. The optimal caste ratio is given as the $r$ at which the total efficiency function has the highest value. In general, this ratio can be obtained as the $r$ at which the differential coefficient of $T(r)$ (represented by $T^{\prime}[r]$ ) becomes 0 . The differential function of $T(r)$ is represented by the equation $T^{\prime}(r)=\alpha_{1}, \alpha_{2}, \alpha_{3}, \ldots \alpha_{t}\left\{t_{1}^{\prime}(r) t_{2}(r) \ldots t_{t}(r)\right.$ $\left.+t_{1}(r) t^{\prime}{ }_{2}(r) \ldots t_{t}(r)+t_{1}(r) t_{2}(r) \ldots t_{t}^{\prime}(r)\right\}$, where $t_{i}^{\prime}(r)$ is the differential coefficient for $t_{i}(r)$ at $r$. Because $0<\alpha_{i} \leq 1$, this function is a proportionally scaleddown version of $T^{\prime}(r)$ in the case when all $\alpha_{i}=1$. Thus, the $r$ that gives the highest value of $T^{\prime}(r)$ is independent from values of $\alpha_{i}$. In addition, the shape of each $t_{i}(r)$ has no effect on the result. Therefore, the optimal caste composition can be obtained when all partial efficiency functions are differentiable.

In empirical studies, the shape of $t_{i}(r)$ can be determined when the quantitative degree of specialization by each caste for each role is known. Therefore, at least in a colony of a dimorphic species, the optimal caste ratio can be estimated from a quantitative assessment of caste specialization, regardless of the shape and relative height of the efficiency function for each task.

\section{APPLICATION TO COLOBOPSIS NIPPONICUS DATA}

\section{The Study Organism}

The model was applied to data obtained from observations of the dimorphic ant Colobopsis nipponicus. The genus Colobopsis was recently separated from the genus Camponotus (Hölldobler and Wilson 1990), and all the Colobopsis species have completely dimorphic subcastes in workers. In C. nipponicus, a major worker is about 2:9 times as heavy as a minor worker (Hasegawa 1993a). This difference can be regarded as the difference in production costs between the two subcastes. Major workers have a truncated head that is used to plug nest entrances (Wilson 1974; Cole 1980; Walker 1984; Walker and Stamps 1986; Hasegawa 1993a); therefore, they are regarded as nest defenders. Major workers also store nutrition as liquid and fat bodies in the gaster (Wilson 1974; Hasegawa 
1993a). Ethograms for Colobopsis'species have demonstrated that over half of the major workers' behavior is regurgitation of stored food, whereas minor workers engage in daily tasks, such as brood care and foraging (Wilson 1974; Cole 1980; Walker 1984; Hasegawa 1993a). Detailed studies of quantitative specialization of major workers have been conducted on C. nipponicus (Hasegawa 1993a, $1993 b$ ). These studies indicated that the major workers can perform their own roles (nest defense and food storage) more efficiently than can the minor workers (the extent of specialization is discussed later).

\section{Efficiency of Food Storage}

Previously I showed (Hasegawa 1993a) that, on average, a major worker of C. nipponicus can store 2.14 times more liquid and 1.68 times more fat on a dry weight basis than can a minor worker. I also demonstrated that the storage ability of both subcastes changes with colony size, while the difference in storage ability between the two castes remains constant with changes in colony size. Thus, considering the size difference between the two subcastes, the amounts of storable liquid and fat in a colony as a whole can be calculated for a colony of a given size with various caste ratios. Changes in caste ratio alter the amounts of stored nutrient in a colony, even when colony size is held constant. Storage in a colony is maximized when the colony contains only major workers because major workers are more efficient with respect to storage than minor workers. Thus, the task efficiency for storage (storage efficiency) of a colony with a given caste ratio can be represented by the proportion of the amount of stored nutrient at that caste ratio relative to the possible maximum storage at a caste ratio of $100 \%$ major workers.

Figure 2 shows changes in storage efficiencies with caste ratios for both liquid and fat. As mentioned previously, the lower the caste ratio, the lower the storage efficiency. However, the storage efficiency does not decrease to 0 at $0 \%$ major workers because the minor workers can store some liquid and fat, albeit less efficiently than can the major workers. Note that the numerical proportion of major workers in the total population of workers is used as the caste ratio in this study. Of course, the caste ratio can also be represented by a weight (investment) ratio between the two castes. In field studies, however, numerical ratios are more easily measured than are weight ratios. Thus, in this article, all efficiency functions are calculated as functions of numerical ratios with reference to the weight difference between the two subcastes.

\section{Efficiency of Defenses}

I demonstrated (Hasegawa 1993b) that most nests without major workers are usurped by conspecific colonies. A single major worker per nest entrance is sufficient to defend the nest effectively from competitors. I also showed that most nests without major workers were usurped or disappeared during the first $2 \mathrm{wk}$ from the start of an experiment, but thereafter the rate of decrease in number of nests did not differ between nests with and without major workers. This observation means that competition for nest site was most intense during the first $2 \mathrm{wk}$. Therefore, the efficiency of defense of a colony was represented by the regression 


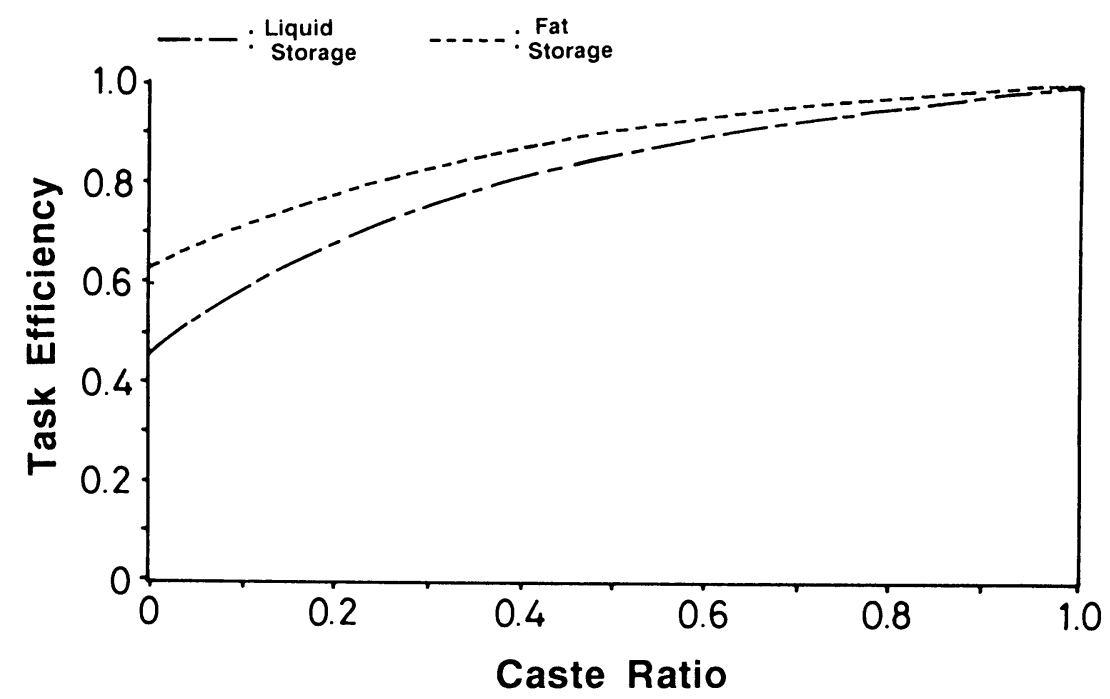

FIG. 2.-Changes in food storage efficiency of a colony of Colobopsis nipponicus. The efficiency curves for both liquid and fat are shown. The size of the colony is assumed to be $200 \mathrm{mg}$. (Data are from Hasegawa 1993a.)

of the survival rate on caste ratio at the $2 \mathrm{wk}$ from the start of the experiment. Because the regression was calculated for log-transformed data, a caste ratio of $0 \%$ was replaced with a dummy variable (0.001). Figure 3 shows the relationship between survival rate and caste ratio in the experimental colonies. This function was used directly to estimate the efficiency of defenses at a given caste ratio.

\section{Efficiency of Performance of Daily Task}

Because major workers of $C$. nipponicus do not engage in daily tasks, such as brood care, care of the queen(s), and foraging (Hasegawa 1993a), performance of such tasks would decrease with caste ratio. Because minor workers perform all daily tasks with the exception of nest defense, the number of minor workers itself should reflect the performance of daily tasks in the colony. Thus, changes in numbers of minor workers can be used as a function of efficiency of daily tasks. Although a major worker is 2.9 times as heavy as a minor worker on average, the difference in size changes with colony size (see Hasegawa 1993a). Thus, shape of the curve representing daily task efficiency changes with colony size. From the pupal dry weights of both subcastes, changes in number of minor workers to caste ratio were calculated for colonies of three different sizes. Figure 4 shows the changes in daily task efficiency with the caste ratio for colonies of three different sizes. Each efficiency curve has a concave shape because the number of minor workers does not decrease linearly with caste ratio as a result of size differences between the two subcastes. Larger colonies have more concave functions, reflecting the fact that the size difference is larger in larger colonies. By using the appropriate daily task efficiency curve for a given colony size, we can estimate the optimal caste ratio more accurately. 


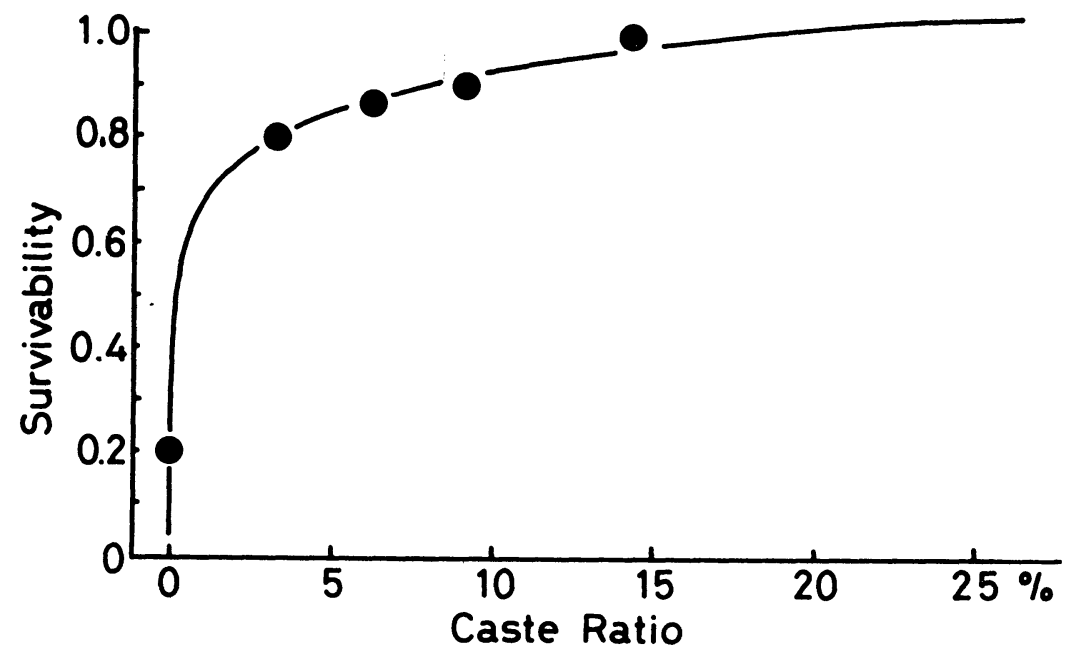

FIG. 3.-Relationship between caste ratio and survival rate of colonies in the field experiment. This curve can be regarded as showing the change in the defensive efficiency of a colony as the caste ratio increases. (Data are from Hasegawa 1993b.)

\section{Estimation of the Optimal Caste Ratio}

As mentioned previously, when changes in the efficiency of performance of each role with caste ratio are known, the total efficiency can be represented by the product of all partial efficiencies at a given caste ratio. Then, the optimal caste ratio can be estimated to be the ratio that gives the maximum total efficiency of the colony as a whole. In calculation, the maximum values of the efficiency function for each task were assumed to be the same, and such an assumption has no effect on the results (see the description of the model). Figure $5 A-C$ shows total efficiency functions based on different assumptions about the roles of the major workers. The efficiency functions in figure $5 A-C$ were calculated for a colony size of $200 \mathrm{mg}$ because this size is typical in the field. When major workers were assumed to perform nest defense only, the maximum value of the total efficiency function was found at a caste ratio of approximately $6 \%$ (fig. $5 \mathrm{~A}$ ). When storage is assumed to be the only role of the major workers, the optimal caste ratio is about $16 \%$ (fig. $5 B$ ). When both storage and defense are included in the model, the optimal caste ratio increased to about $20 \%$ (fig. $5 \mathrm{C}$ ). The former two cases are unlikely, but they do show that the model can incorporate each function of the subcastes independently.

\section{Comparison with the Observed Distribution of Caste Ratio in the Field}

The optimal caste ratios, based on the prior three assumptions, were calculated for a range of colony sizes $(0-800 \mathrm{mg})$ and are represented as a function of colony size. As mentioned previously, the daily task efficiency curves are more concave for larger colonies; thus, the optimal caste ratio is slightly lower in larger colonies than in smaller colonies. The estimated optimal ratio functions (the solid lines in 


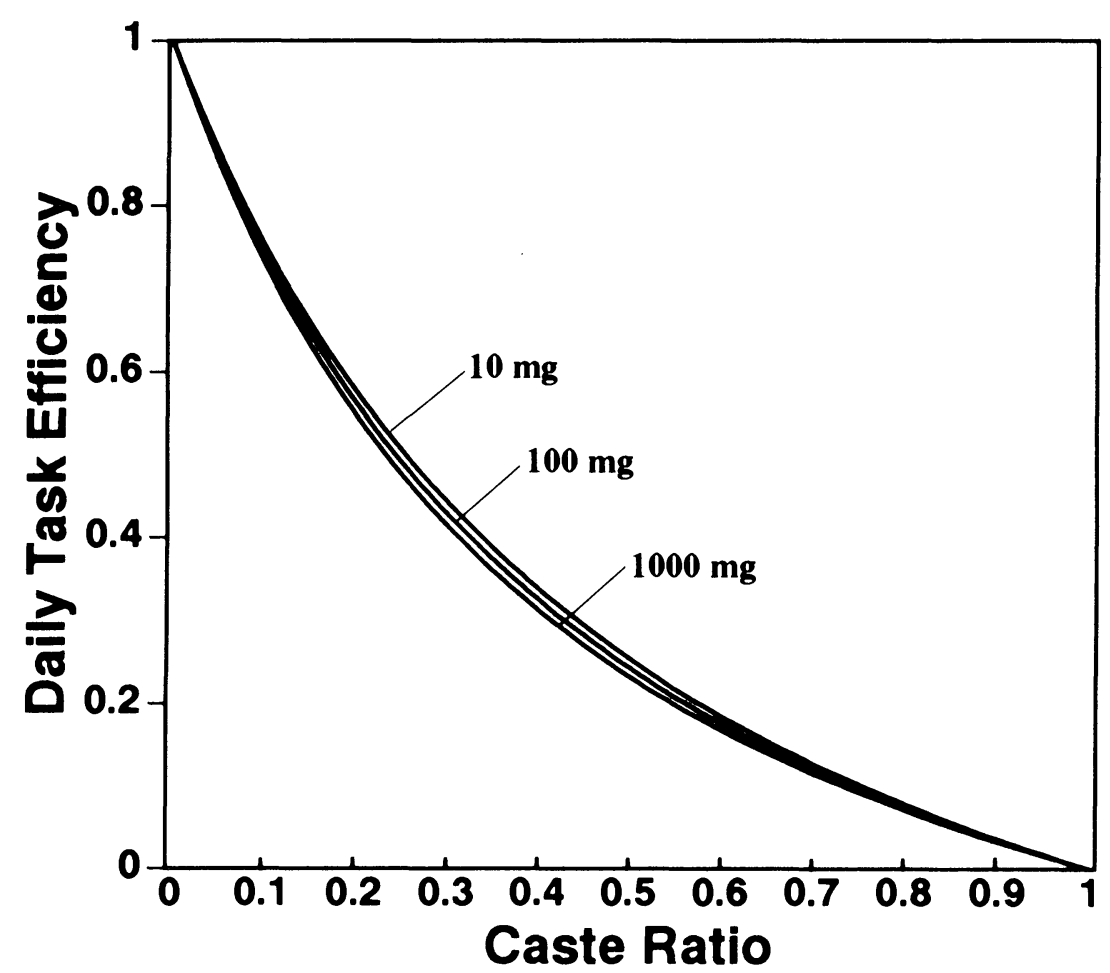

FIG. 4.-Changes in daily task efficiency in a colony of Colobopsis nipponicus. It is assumed that the efficiency of performance of daily tasks, such as foraging, brood care, and nest maintenance, is proportional to the number of minor workers in the colony.

fig. $6 A-C$ ) are shown together with the observed distribution of caste ratio in 196 colonies collected in the field from June 1990 to October 1991. In each panel, dotted lines represent the ratios that yield $95 \%$ of the optimal efficiency value. When only defense or storage was included in the model, the estimated ratio function did not fit well with the observed distribution of caste ratios (fig. $6 A, B$ ). However, when both defense and storage were included in the model, the estimated curve was in a good agreement with the observed distribution of ratios (fig. $6 \mathrm{C}$ ). In addition, caste ratios of most field colonies fell within a range that gave more than $95 \%$ of the optimal efficiency.

\section{Experimental Manipulation of the Caste Ratio}

To examine whether changes in caste ratio actually affect brood production, laboratory colonies were experimentally manipulated. Setup conditions for the experiment are shown in table 1. Five experimental treatments with five replicates each were examined using adults and larvae from reared colonies. Each experimental colony consisted of $100 \mathrm{mg}$ of workers with $0 \%, 10 \%, 20 \%, 30 \%, 50 \%$, or $100 \%$ of major workers in numbers. Workers in each colony were given 100 larvae in larval classes 1-3 (the larvae do not attain size of pupation; see 

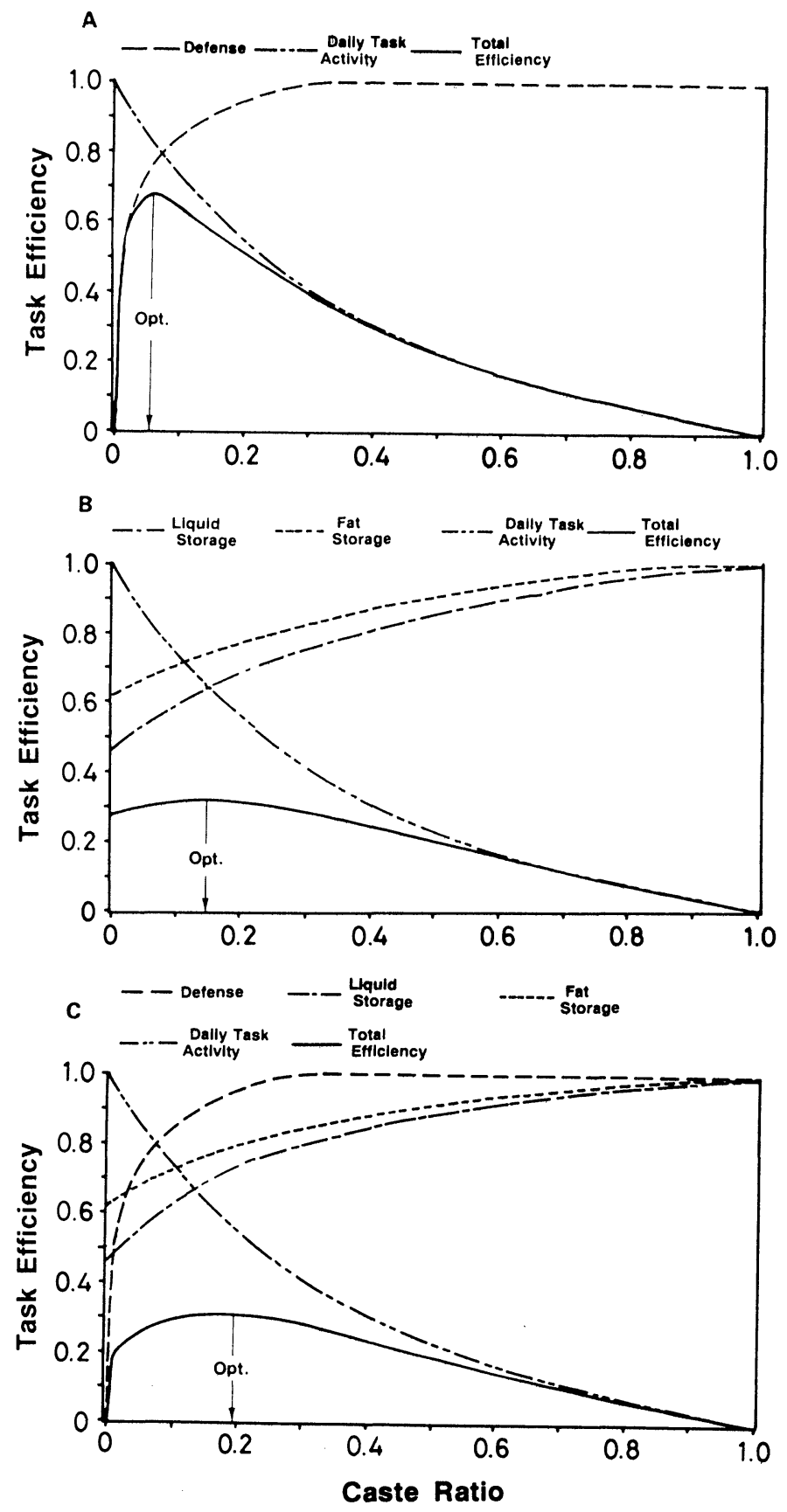

FIG. 5.-Estimation of the optimal caste ratio based on three different assumptions for the roles of the major workers. In figure $5 A$, only defense is considered as the majors' role. In figure $5 B$, only food storage is considered as the majors' role. Figure $5 C$ includes effects of both nest defense and food storage. 

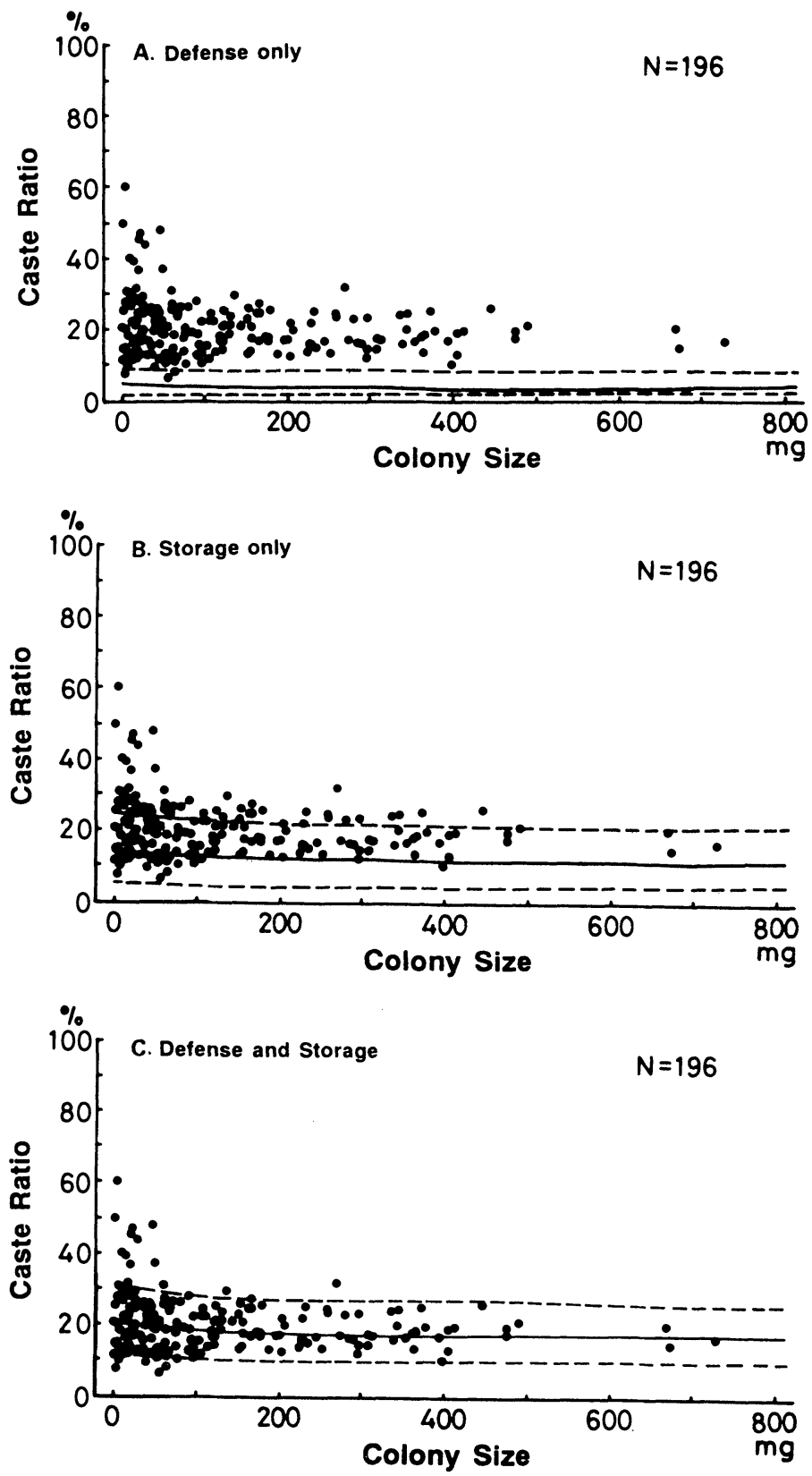

FIG. 6. - Comparison of the estimated ratios with the observed distribution of caste ratios in 196 field colonies. Figure $6 A-C$ corresponds to the three assumed roles of majors: defense only, storage only, and defense plus storage. The solid line represents the estimated optimal ratios. The dotted lines represent ratios that are at $95 \%$ efficiency of the optimal ratio. 
TABLE 1

Setup Condition and Results of the Caste Ratio Manipulation Experiment

\begin{tabular}{|c|c|c|c|c|c|c|}
\hline \multirow{2}{*}{$\begin{array}{l}\text { Caste Ratio } \\
(\%)\end{array}$} & \multirow{2}{*}{$\begin{array}{l}\text { NUMBER OF } \\
\text { MiNORS }\end{array}$} & \multirow{2}{*}{$\begin{array}{l}\text { Number oF } \\
\text { MAJORS }\end{array}$} & \multicolumn{2}{|c|}{$\begin{array}{l}\text { Total Live Weight of } \\
\text { Larvae (mEAN } \pm \text { SD) } \\
(\mathrm{mg})\end{array}$} & \multirow{2}{*}{$\begin{array}{c}\text { Number of } \\
\text { Surviving LaRvae }\end{array}$} & \multirow[b]{2}{*}{$N$} \\
\hline & & & Initial & Final & & \\
\hline 0 & 100 & 0 & $62.0 \pm 2.3$ & $58.9 \pm 8.8$ & $54.2 \pm 7.5$ & 5 \\
\hline 10 & 76 & 8 & $61.3 \pm 1.6$ & $70.0 \pm 5.9$ & $61.1 \pm 10.8$ & 5 \\
\hline 20 & 57 & 14 & $62.3 \pm 2.7$ & $77.1 \pm 5.2$ & $58.0 \pm 2.5$ & 5 \\
\hline 30 & 44 & 19 & $63.1 \pm 2.9$ & $71.0 \pm 3.9$ & $63.6 \pm 7.0$ & 5 \\
\hline 50 & 25 & 25 & $61.5 \pm 1.1$ & $67.6 \pm 3.2$ & $62.6 \pm 9.6$ & 5 \\
\hline 100 & 0 & 34 & $61.6 \pm 2.4$ & $68.6 \pm 3.1$ & $61.1 \pm 3.2$ & 5 \\
\hline
\end{tabular}

NotE.-Each colony was cultured for $1 \mathrm{mo}$, and the initial number of larvae was 100 in each case.

TABLE 2

Statistical Tests for Results of the Caste Ratio Manipulation

\begin{tabular}{|c|c|c|c|c|c|c|}
\hline \multirow{2}{*}{$\begin{array}{l}\text { EXPERIMENTAL } \\
\text { TREATMENT (\%) }\end{array}$} & \multicolumn{6}{|c|}{ EXPERIMENTAL TREATMENT (PROPORTION OF MAJORS) } \\
\hline & $0 \%$ & $10 \%$ & $20 \%$ & $30 \%$ & $50 \%$ & $100 \%$ \\
\hline 0 & & -1.262 & -1.082 & -2.056 & -1.547 & -2.037 \\
\hline 10 & $-2.569^{*}$ & & .728 & -.348 & -.155 & -.000 \\
\hline 20 & $-4.218^{*}$ & -2.007 & & -1.692 & -1.040 & -1.994 \\
\hline 30 & $-3.052 *$ & -.297 & -2.108 & $\ldots$ & .189 & .582 \\
\hline 50 & $-2.342^{*}$ & -.785 & $3.444 * *$ & 1.461 & & .221 \\
\hline 100 & $-2.580^{*}$ & .475 & $3.128^{*}$ & 1.057 & -.472 & $\ldots$ \\
\hline
\end{tabular}

NorE.-The values in the upper-right corner show $t$ values for numbers of surviving larvae, and those in the lower-left corner are $t$ values for total weights of surviving larvae.

$* P<.05$.

** $P<.01$.

Hasegawa 1992 for details of the classes) at the onset of the experiment. The number of larvae in each size class was equalized in each colony to give an equal total live weight of larvae to each colony. Each experimental colony was housed in a hollow bamboo stem and reared under constant conditions $\left(25^{\circ} \mathrm{C}, 60 \%\right.$ relative humidity, 14L:10D). The colonies were supplied ad lib. with an artificial ant diet (Bhatkar and Whitcomb 1970) in liquid form. One month later, the numbers of surviving larvae and their total live weights were recorded.

A statistical test showed that the average number of surviving larvae did not differ between any pair of treatments. However, the total larval weight at the end of the experiment was significantly different between several pairs (table 2). Because the number of surviving larvae did not differ between the treatments, differences in the total larval weight represent the weight gain per larva in each treatment and thus reflect the ergonomic efficiency of the colony as a whole. The increments in total larval weight for each treatment are shown in figure 7 . The group with a caste ratio of $20 \%$ showed the largest incremental value among all 


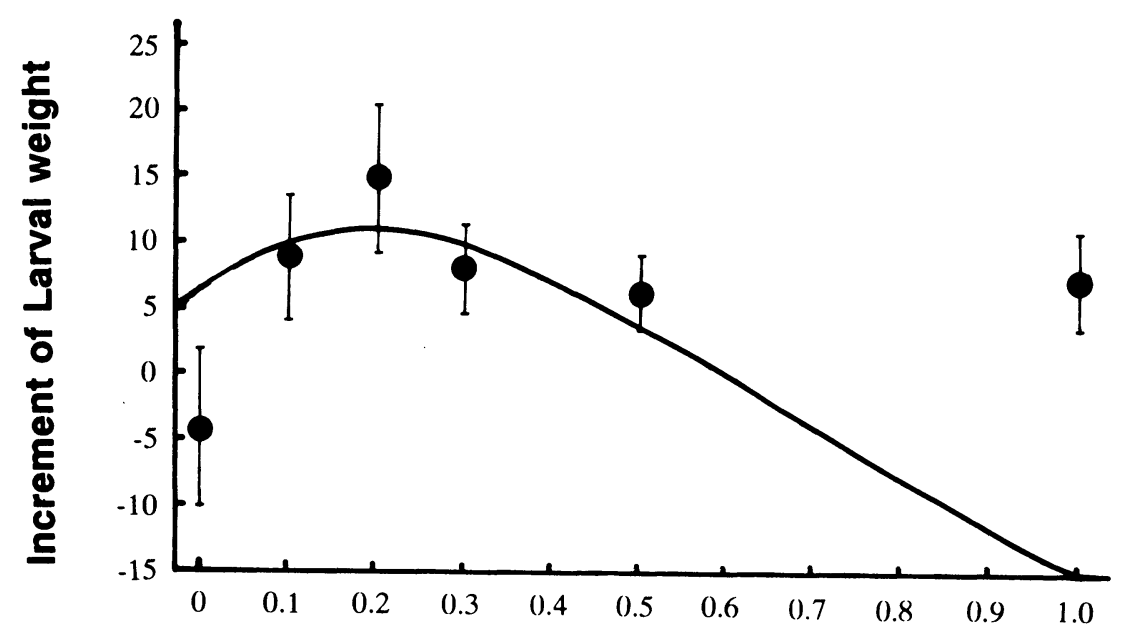

Caste Ratio

FIG. 7.-Increments in total larval weight for experimental colonies with different caste ratios. The means with $95 \%$ confidence limits are shown. The superimposed curve is the same line as the total efficiency function in figure $5 B$. This line was obtained by assuming that storage was the majors' only role since the possibility of competition for a nest site was eliminated from the experiment.

treatment groups. Thus, the group with $20 \%$ major workers operated with the highest ergonomic efficiency of all the groups.

\section{DISCUSSION}

The quantitative analysis of task efficiency showed that the ergonomic efficiency of the colony for storage or defense should increase, while that for daily tasks should decrease with caste ratio (see also Hasegawa 1993a, 1993b). These effects of caste ratio on the colony's efficiency for different tasks should result in a specific caste ratio at which the colony maximizes its reproductive output. Thus, the optimal caste ratio can be estimated by applying the model described in this article. When only storage or defense was included in the model, the estimated caste ratio was lower than the observed ratio. These conditions are unlikely because the major workers of Colobopsis nipponicus actually specialize in both tasks. Thus, disagreements of the observed ratios with the estimates are reasonable. When both tasks were included in the model, the estimated ratio was consistent with the observed ratios, which suggests that the caste ratio of $C$. nipponicus is tuned via interactions between a colony's efficiency with respect to all three tasks and that the model is useful for estimating the optimal caste ratio.

The major workers of $C$. nipponicus specialize in two different roles, storage of nutrition and nest defense (Hasegawa 1993a, 1993b). Even a single major can effectively defend a nest, but colonies without majors do not survive (Hasegawa 
$1993 b$ ). Thus, it appears that a few majors (at least as many as there are nest entrances) are required for defense, but a large number of majors would be a waste of the colony's resources because, even when a colony becomes very large, there are usually fewer than 20 nest entrances (E. Hasegawa, unpublished data). However, large colonies often contain more than 200 majors (E. Hasegawa, unpublished data) - in other words, considerably more than the minimum number needed for nest defense. Therefore, the observed caste ratio in field colonies (ca. $20 \%$ ) suggests the importance of storage of nutrition in this species.

Evidence from other studies supports this conclusion. The termite Cryptotermes domesticus has a soldier caste with similar head morphology to that of majors of $C$. nipponicus, and soldiers engage in nest defense by head plugging (E. Hasegawa, personal observation). Because $C$. domesticus nests in dry dead wood and eats the nest material itself, it is hard to assume a storage role for soldiers. Thus, the present model predicts a few soldiers among all colony members if the soldiers are highly effective in defending nest and less effective performing daily tasks (these assumptions are quite plausible). The soldiers of $C$. domesticus represented only $1 \%-2 \%$ of the total colony members in nests on the Bonin Islands in southern Japan (E. Hasegawa, personal observation). Thus, the importance of nutrition storage might be responsible for the higher caste ratio in C. nipponicus.

The caste manipulation experiment showed that the colonies with a caste ratio of $20 \%$ reared larvae more efficiently than did other colonies. This result strongly suggests that the ergonomic efficiency of the colony is actually affected by the caste ratio. Porter and Tschinkel (1985) showed that polymorphic colonies of Solenopsis invicta produced broods more efficiently than artificial monomorphic colonies. Among the social insects, it is well known that individuals of different ages show different behaviors, namely, age polyethism or an age-based caste (see Hölldobler and Wilson 1990). In the morphologically monomorphic ant Pristomyrmex pungens, when age-caste ratios were altered, colonies with different caste ratios showed different brood productivities (Tsuji 1994). These studies suggest the regulation of colony efficiency through the caste ratio, even in the case of age-based castes in monomorphic species. As previously discussed, the present model can be extended to polymorphic species that have more than two castes, based on either age or morphology. Therefore, the model presented here should be useful for estimations of the adaptive demography among subcastes when the degree of specialization of each subcaste to each role can be assessed quantitatively.

In the caste manipulation experiment, $20 \%$ majors was the most efficient ratio for brood production. However, the result must be interpreted with caution. The possibility of competition for nesting site was eliminated from the experiment. Thus, the total efficiency function was expected to be different from that of the field colonies. In the present case, the total efficiency function should be a synthesized function that incorporates storage and daily task efficiencies. Thus, the predicted function should have a similar shape to that of the total efficiency function in figure $5 B$. The total efficiency function in figure $5 B$ was superimposed on the data in figure 7 . The superimposed curve has a peak at a ratio of about 
$16 \%$ but still fits well with the observed productivity, except at a caste ratio of $100 \%$ majors (the reasons for this disagreement are discussed later). Thus, in spite of the differences between experimental and field conditions, the results match the predictions of the model.

Why was the observed productivity at a ratio of $100 \%$ not consistent with the predicted value? It is well known that a caste that specializes in specific tasks can perform other roles on some occasions, a phenomenon known as behavioral plasticity. Wilson (1985) showed that in the dimorphic ant Pheidole pubiventris, majors' activity for daily tasks increased dramatically when they exceeded $80 \%$ of the total worker population. Thus, the high productivity at the caste ratio of $100 \%$ in the present experiment might be explained by behavioral plasticity of $C$. nipponicus majors. However, behavioral plasticity should not have a major effect on the results of the experiment in this study because majors generally do not engage in daily tasks until they attain a high proportion (over $80 \%$ in Wilson's study). Thus, the observed differences in brood productivity at lower caste ratios would reflect the differences in ergonomic efficiency predicted by the model.

Caste ratio is a characteristic that is expressed at the colony level. Thus, the most efficient caste ratio, or, more precisely, a worker's ability to regulate the caste composition as the optimal ratio, is favored through natural selection that works on the colony as a whole because maximization of the colony's ergonomic efficiency contributes equally to the fitness of all workers. Of course, if there are both fertile and sterile workers concerning male production, evolutionary interest in reproductive output of the colony as a whole might differ between the two groups. There would be, however, no intracolonial conflict between workers in C. nipponicus because all workers do not lay eggs in the presence of a queen (Hasegawa 1994). In addition, if a conflict arises over the sex ratio between the queen and the workers (see Trivers and Hare 1976; this is indeed the case in $C$. nipponicus; see Hasegawa 1994), the high productivity of the colony is favorable to both parties. Thus, by studying caste ratios in social insects, we can characterize the hierarchical selection structure that operates on social groups.

Lets us discuss the general predictions and limitations of the present model. The model allows us to estimate the most efficient caste composition when the quantitative specialization of each caste to different tasks is known. Thus, the model is useful for studies of colony-level selection through the caste ratio and its interactions with individual selection. Moreover, the model makes some general predictions about caste composition. When there is little size difference among castes, the model predicts that the proportion of a caste will be high when this caste is highly specialized for performance of a specific task. This prediction is easily understood when we compare figure $1 A$ and $B$. If a caste is highly specialized in a task, the efficiency function of the caste has a steep slope, and the optimal proportion of the caste is high (fig. 1A). However, the efficiency function of a less specialized caste has a gentler slope, and the optimal proportion is low (fig. $1 B$ ). Nevertheless, this prediction does not hold when there is a large size difference between castes or when the effects of tasks on colony efficiency are not additive. In these cases, the efficiency function changes nonlinearly with the numerical proportion of the caste (as in fig. $1 C$ ). In such cases, the general ten- 
dency in the optimal caste proportions cannot be predicted from degree of specialization only.

One limitation of the model is that it makes no predictions about the causes and the direction of specialization of castes. The model can estimate the optimal caste composition, based on the present degree of specialization, but it cannot identify evolutionary factors that originally promoted the differentiation of castes. The main cause of the evolution of the worker subcastes is still unknown. In a consideration of proximate mechanisms of subcaste differentiation, Wheeler (1991) discussed the developmental basis of worker polymorphism in ants and suggested that alternative phenotypes of the same genotype might promote caste differentiation. Morphological polymorphism might appear from a single mutation in genes that regulate the developmental schedule of larvae. However, worker subcastes can occur without genetic differentiation among castes. In fact, under experimental conditions, larvae of $C$. nipponicus can develop into both queens and major workers depending on the nutritional conditions of the colony (E. Hasegawa, unpublished data). Thus, alternative phenotypes of the same genotype have to be considered in studies of caste differentiation. The model presented here has little power in such interesting studies. However, the model does help us elucidate hierarchical selection structures on social groups, which are issues of major importance in evolutionary ecology.

\section{ACKNOWLEDGMENTS}

I thank K. Miyashita and T. Kusano for many helpful suggestions and for their encouragement during the course of this study and E. O. Wilson for his comments on the early draft. I acknowledge L. Frenette and J. Frenette for improvements in English expressions. I also thank the associate editor of the American Naturalist and two anonymous reviewers for their critical comments on the manuscript.

\section{LITERATURE CITED}

Bhatkar, A., and W. H. Whitcomb. 1970. Artificial diet for rearing various species of ants. Florida Entomologist 53:229-232.

Busher, C. E., P. Calabi, and J. F. A. Traniello. 1985. Polymorphism and division of labor in the Neotropical ant Camponotus sericeiventris Guerin (Hymenoptera: Formicidae). Annuals of Entomological Society of America 78:221-228.

Cole, B. J. 1980. Repertoire convergence in two mangrove ants, Zacryptocerus varianus and Camponotus (Colobopsis) sp. Insectes Sociaux 27:265-275.

Detrain, C., and J. M. Pasteels. 1992. Caste polyethism and collective defense in the ant, Pheidole pallidura: the outcome of quantitative differences in recruitment. Behavioral Ecology and Sociobiology 29:405-412.

Fowler, H. G. 1987. Worker polymorphism in field colonies of carpenter ants (Hymenoptera: Formicidae: Camponotus): stochastic selection? Insectes Sociaux 34:204-210.

Hasegawa, E. 1992. Annual life cycle and timing of male egg production in the ant Colobopsis nipponicus (Wheeler). Insectes Sociaux 39:439-446.

1993a. Caste specialization in food storage in the dimorphic ant Colobopsis nipponicus (Wheeler). Insectes Sociaux 40:261-271. 
1993b. Nest defense and early production of the major workers in the dimorphic ant Colobopsis nipponicus (Wheeler) (Hymenoptera: Formicidae). Behavioral Ecology and Sociobiology 33:73-77.

Hölldobler, B., and E. O. Wilson. 1990. The ants. Harvard University Press, Belknap, Cambridge, Mass.

Lachaud, J. P., L. Passera, A. Grimal, C. Detrain, and G. Beugnon. 1992. Lipid storage by major workers and starvation resistance in the ant Pheidole pallidula (Hymenoptera, Formicidae). Pages 153-160 in J. Billen ed. Biology and evolution of social insects. Leuven University Press, Leuven, Belgium.

Mirenda, J. T., and S. B. Vinson. 1981. Division of labor and specification of castes in the red imported fire ant Solenopsis invicta Buren. Animal Behaviour 29:410-420.

Moffet, M. W. 1986. Notes on the behavior of the dimorphic ant Oligomyrmex overbecki (Hymenoptera: Formicidae). Psyche 93:107-116.

1987. Division of labor and diet in the extremely polymorphic ant Pheidologeton diversus. National Geographic Research 3:282-304.

Oster, G. F., and E. O. Wilson, 1978. Caste and ecology in the social insects. Princeton University Press, Princeton, N.J.

Patel, A. D. 1990. An unusually broad behavioral repertory for a major worker in a dimorphic ant species: Pheidole morrisi (Hymenoptera: Formicidae) Psyche 97:181-191.

Porter, S. D., and W. R. Tschinkel. 1985. Fire ant polymorphism (Hymenoptera: Formicidae): factors affecting worker size. Annuals of Entomological Society of America 78:381-386.

Tsuji, K. 1990. Nutrient storage in the major workers of Pheidole ryukyuensis (Hymenoptera: Formicidae). Applied Entomology and Zoology 25:283-287.

1994. Inter-colonial selection for the maintenance of cooperative breeding in the ant Pristomyrmex pungens: a laboratory experiment. Behavioral Ecology and Sociobiology 35: 109-113.

Villet, M. H. 1990. Division of labor in the Metabele ant Megaponera foetens (Fabr.) (Hymenoptera: Formicidae). Ethology, Ecology \& Evolution 2:397-417.

Walker, J. M. 1984. The soldier caste and colony structure in the ant Camponotus (Colobopsis) impressus. Ph.D. diss. University of California, Davis.

Walker, J. M., and J. Stamps 1986. A test of optimal caste ratio theory using the ant Camponotus (Colobopsis) impressus. Ecology 67:1052-1062.

Wheeler, D. E. 1991. The developmental basis of worker caste polymorphism in ants. American Naturalist 138:1218-1238.

Wilson, E. O. 1953. The origin and evolution of polymorphism in ants. Quarterly Review of Biology 28:136-156.

1971. The insect societies. Harvard University Press, Belknap, Cambridge, Mass.

1974. The soldier of the ant Camponotus (Colobopsis) fraxinicola as a trophic caste. Psyche 81:182-188.

1976. A social ethogram of the Neotropical arboreal ant Zacryptocerus varianus (Fr. Smith). Animal Behaviour 34:354-363.

1978. Division of labor in fire ants based on physical castes (Hymenoptera: Formicidae: Solenopsis). Journal of Kansas Entomological Society 51:615-636.

1980a. Caste and division of labor in leaf-cutter ants (Hymenoptera: Formicidae: Atta). I. The overall pattern in $A$. sexdens. Behavioral Ecology and Sociobiology 7:143-156.

1980b. Caste and division of labor in leaf-cutter ants (Hymenoptera: Formicidae: Atta). II. The ergonomic optimization of leaf cutting. Behavioral Ecology and Sociobiology 7:157-165. 1985. Between-caste aversion as a basis for division of labor in the ant Pheidole pubiventris (Hymenoptera: Formicidae). Behavioral Ecology and Sociobiology 17:35-37.

Associate Editor: Richard D. Howard 